\title{
Corrigendum: Is adolescent e-cigarette use associated with smoking in the United Kingdom?: A systematic review with meta-analysis
}

\author{
Adewale Aladeokin', Catherine Haighton'
}

\section{Corrigendum on:}

Is adolescent e-cigarette use associated with smoking in the United Kingdom?:

A systematic review with meta-analysis

By Adewale Aladeokin, Catherine Haighton

Tobacco Prevention and Cessation, Volume 5, Issue April, Pages 1-13

Publish date: 22 April 2019

DOI: https://doi.org/10.18332/tpc/108553

An error in data entry occurred during the production of Figure 3 in the manuscript, as the authors accidentally entered the published adjusted ORs from three included studies as Log Odds in the generic inverse variance meta-analysis. Correcting the error shows that the OR remains significant: $3.86 ; \mathrm{p}<0.00001$ instead of $26.01[5.35,126.44]$. The conclusions were based on the meta-analysis of unadjusted ORs which was higher than this (OR: 5.55). However, as people are reportedly dying from severe lung - related illnesses tied to e-cigarettes and many countries are banning e-cigarettes it remains important to review the evidence that is currently available.

\section{AFFILIATION}

1 Northumbria University, Newcastle, United Kingdom

CORRESPONDENCE TO Catherine Haighton. Northumbria University, Newcastle, United Kingdom. E-mail: katie.haighton@ northumbria.ac.uk ORCID ID: https://orcid.org/0000-0002-80610428

\section{KEYWORDS}

adolescent, e-cigarette, United Kingdom, systematic review, smoking

Received: 5 November 2019 Accepted: 5 November 2019

Figure 3: Meta-analysis based on adjusted odds ratios

The figure is the following:

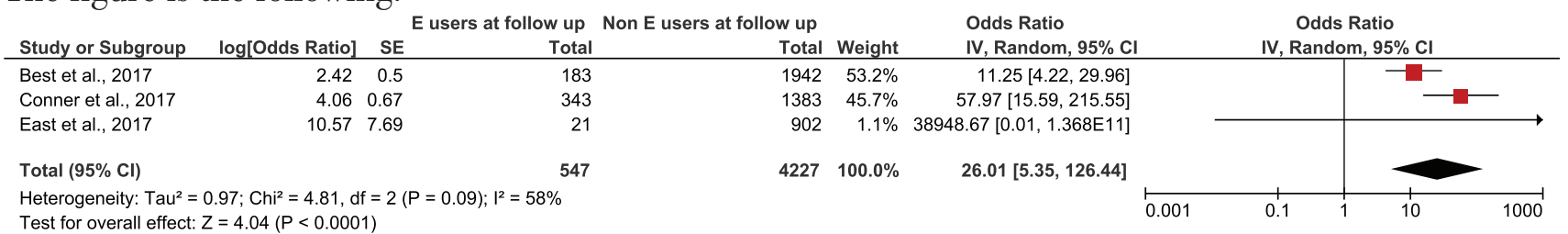

The correct figure should be:

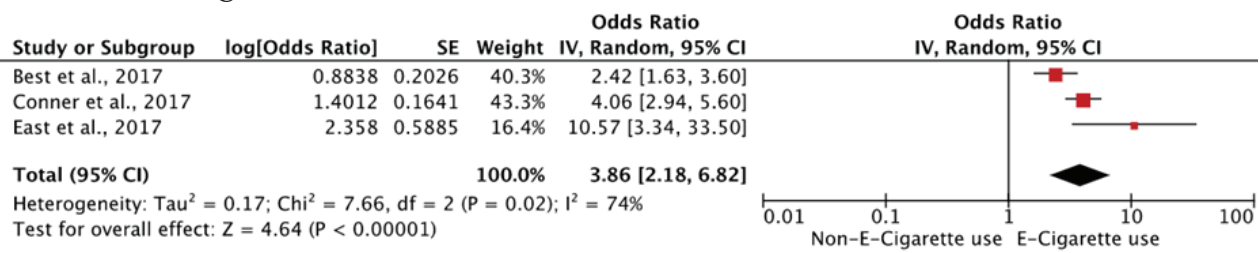

